\title{
Matrix-valued wave packet Bessel sequences and symmetric frames in $L^{2}\left(\mathbb{R}^{d}, \mathbb{C}^{s \times r}\right)$
}

\author{
JYOTI, LALIT K. VASHISHT ${ }^{\dagger}$, GEETIKA VERMA AND VIRENDER
}

\author{
Date of Receiving : $\quad 06.04 .2018$ \\ Date of Revision : $\quad 21.07 .2018$ \\ Date of Acceptance : 22.07 .2018
}

\begin{abstract}
We consider matrix-valued wave packet systems in the matrix-valued function space $L^{2}\left(\mathbb{R}^{d}, \mathbb{C}^{s \times r}\right)(d, s, r \geq 1)$. Some results on matrix-valued wave packet Bessel sequences have been extensively discussed in view to generate frames from Bessel sequences in $L^{2}\left(\mathbb{R}^{d}, \mathbb{C}^{s \times r}\right)$. Necessary conditions for matrix-valued Bessel sequences in terms of an estimate of a series related to the Fourier transform of the matrix-valued wave packet function are given. The frame property of a matrix-valued symmetric wave packet function in higher dimensions is discussed.
\end{abstract}

\section{Introduction}

Antolín and Zalik in [1], introduced and studied matrix-valued wavelets for the matrix-valued function space $L^{2}\left(\mathbb{R}^{d}, \mathbb{C}^{n \times n}\right)$. The matrix-valued function space $L^{2}\left(\mathbb{R}^{d}, \mathbb{C}^{n \times n}\right)$ is related to video imaging. Xia and Suter $[29]$ classified and constructed vector-valued (matrix-valued) wavelets with sampling property. They showed that certain linear combinations of known scalar-valued wavelets may yield multiwavelets. Inspired by the work in [1], Jyoti, Deepshikha, Vashisht and Verma [18] studied matrixvalued wave packet frames in $L^{2}\left(\mathbb{R}^{d}, \mathbb{C}^{s \times r}\right)$, where $s$ and $r$ are positive integers. They discussed an interplay between wave packet frames in the Lebesgue space $L^{2}\left(\mathbb{R}^{d}\right)$ and matrix-valued wave packet frames for the function space $L^{2}\left(\mathbb{R}^{d}, \mathbb{C}^{s \times r}\right)$. The first purpose of this paper is to show some relation between the series related to the Fourier

2010 Mathematics Subject Classification. 42C15, 42C30, 42C40, 43A32.

Key words and phrases. Frame, Bessel sequence, symmetric frames, wave packet.

The research of Jyoti is supported by the Council of Scientific \& Industrial Research, India (Grant No.: 09/045(1374)/2015-EMR-I). Some part of this paper was prepared at the International Workshop on Wavelets, Frames and Applications (IWWFA)-III (2017), Delhi, during December 14-20, 2017.

We would like to thank the referee for reading the paper carefully and valuable comments towards the improvement of the paper.

Communicated by: Khole Timothy Poumai

${ }^{\dagger}$ Corresponding author. 